\title{
ANÁLISIS DE LA HABILIDAD COMUNICATIVA DOCENTE Y PAUTAS DE ACTUACIÓN. ATRAER LA ATENCIÓN HABLANDO: UN RETO PARA LA ENSEÑANZA UNIVERSITARIA
}

\author{
José Antonio Barrio del Campo \\ Departamento de Educación. Universidad de Cantabria \\ barrioja@unican.es \\ Ángela Barrio Fernández \\ IES. Santa Clara. Santander
}

Fecha de Recepción: 10 Enero 2018

Fecha de Admisión: 10 Abril 2018

\section{RESUMEN}

Desde el ámbito educativo universitario es indudable que la comunicación representa, hoy más que nunca, un tema cardinal. El hecho de que sobre una Comunicación Eficaz se sustenta en gran medida la calidad educativa, es una realidad insoslayable (Barrio y Borragán., 2005). En el contexto del EEES, el docente deberá asumir una serie de cambios sustanciales en la metodología de enseñanza aprendizaje, en las habilidades y competencias que deberá desarrollar dentro y fuera del aula, así como la incorporación de nuevos modelos pedagógicos en relación con la comunicación en clase (Barrio y Borragán, 2011).

Conocemos mucho sobre algunas cuestiones importantes para la comunicación eficaz, sin embargo, ¿conocemos los indicadores más eficaces y las pautas precisas para el trabajo de esta habilidad entre los docentes?. Parece que sigue siendo conveniente el desarrollo de instrumentos de análisis de esta habilidad. En este sentido, el instrumento con el que se ha trabajado, (herramienta para el Análisis de la Habilidad Comunicativa Docente) incluye nueve dimensiones básicas en la habilidad comunicativa de la función docente: mensaje; atención, comprensión y feedback; lenguaje; habla, voz y lenguaje corporal; acción y experimentación; inducción al trabajo, al estudio y a la próxima sesión de clase; participación de los alumnos; personalidad, empatía, seducción y optimismo; y relaciones del profesor con el estudiante.

Hemos contado con la colaboración a través de su cumplimentación de 156 docentes universitarios, de diferentes disciplinas. Los resultados que se están obteniendo, nos están permitiendo seguir trabajando para ofrecer pautas de actuación cada vez más específicas en relación con la mejora de la habilidad comunicativa del docente universitario.

Palabras clave: comunicación; habilidad comunicativa; enseñanza universitaria; evaluación; docencia 


\author{
ABSTRACT \\ Analysis of teaching communicative skill and action guidelines. attract attention talking: a \\ challenge for university teaching.
}

From the university educational field it is undoubted that communication represents, today more than ever, a theme of great importance. The fact that educational quality is largely based on an Effective Communication is an unavoidable reality (Barrio and Borragán., 2005). In the context of the EHEA, the teacher must assume a series of substantial changes in the teaching-learning methodology, as well as in the skills and competences that must be developed inside and outside the classroom, as in the incorporation of new pedagogical models in relation to communication in class (Barrio and Borragán, 2011).

We know a lot about some of the important issues for effective communication. However, do we know the most effective indicators and the precise guidelines for the work of this skill among teachers? It seems that the development of instruments for the analysis of this skill is still appropriate. In this sense, the Loupe of the Aula de Oratoria of the University of Cantabria, that has been used in this work, (an instrument for the Analysis of the Communicative Teaching Skill) includes nine basic dimensions in the communicative ability of the teaching function: message; attention, understanding and feedback; language; speech, voice and body language; action and experimentation; induction to work, to study and to the next class session; participation of students; personality, empathy, seduction and optimism; and teacher's relations with the student.

We have counted on the collaboration of 156 university teachers from different disciplines, through completion of the tool. The results analyzed in this article allow us to continue working to offer increasingly specific action guidelines in relation to improving the communicative ability of the university teacher.

Keywords: communication; communicative ability; university teaching; evaluation; teaching.

\title{
INTRODUCCIÓN
}

Saber comunicar lo que uno conoce o lo que siente es muy importante en la sociedad en la que vivimos. Una gran parte de las profesiones requieren de la palabra como instrumento imprescindible de trabajo, por lo que quien posee una depurada técnica de comunicación tendrá un éxito más probable en todos los aspectos: laborales, científicos, docentes, sociales y personales. Poseer una gran capacidad de comunicación es un valor añadido de cualquier persona y mejora la imagen de la Institución que representa.

La comunicación, tanto desde la vertiente del habla (atraer hablando) como desde la vertiente de la escucha (escuchar con empatía), es una habilidad que debe ser aprendida o mejorada por todos los profesionales y el público en general. Sabemos que si se mejora la capacidad de comunicación y se enseña a mirar al que está enfrente, ello incidirá positivamente en el clima social, el aprendizaje o la cordialidad.

Como la mayoría de las actividades humanas de las que se obtiene un beneficio y son útiles, el modelo de comunicación eficaz con el que venimos trabajando, crea un efecto cascada de aprendizaje y de uso, por lo que a largo plazo se irán viendo los resultados, y en ese camino estamos los implicados en este proyecto. Aunque, los resultados no son inmediatos el objetivo consideramos que es muy provechoso para nuestra sociedad.

En la actualidad, la valoración de la competencia oral en distintos ámbitos sociales y profesionales es cada día mayor (Abascal, 2010; Benavides, 2008; Borragán, Barrio y Borragán, 2009). Sin embargo, la realidad diaria nos muestra muchas veces personas con miedo, con dificultades para hablar en público, a las que les falta poder de convicción, incapaces de transmitir con eficacia y entusiasmo sus pensamientos, sus conocimientos y/o sus sentimientos. Entre los universitarios no es 
infrecuente encontrarnos con problemas de comunicación, bien por desconocimiento de las claves de la oratoria eficaz o bien por causas asociadas a la personalidad, a la forma de estructurar los mensajes 0 a sus actitudes ante la comunicación.

En el caso de los docentes universitarios, son múltiples las situaciones en las que deben de poner en juego sus habilidades comunicativas (p.ej. docencia cotidiana, exposición de trabajos en congresos y reuniones científicas, concursos para la obtención de plazas, proyectos docentes, defensa de argumentos, defensa de una tesis doctoral, entre otras muchas). En la interacción docente y en la comunicación de la ciencia con fascinación, tendremos que ser capaces de suscitar una variedad de deseos, intereses e inquietudes que hagan ilusionarse a quienes nos escuchen y que les motive hacia la acción. Los profesores se ven obligados a modificar sus formas de trabajo hacia un encuentro con las nuevas pautas y ejes pedagógicos (Cifuentes, 2008; Rodríguez, 2007), poniéndose de relieve la importancia de la habilidad comunicativa. El profesor actual deberá conocer y ser capaz de transmitir a sus alumnos los conocimientos de la forma más sugestiva posible, intentando seducirlos para su implicación en el estudio de la asignatura. Para conseguirlo, posiblemente la habilidad más importante mostrada por los profesores sea la "habilidad comunicativa" de forma que atraiga la atención y estimule el pensamiento.

El comienzo de la Configuración del Espacio Europeo de Educación Superior (EEES), junto con las Declaraciones de la Sorbona (1998) y Bolonia (1999), marcaron las líneas maestras para la construcción de la Europa del Conocimiento, en la que la investigación, la innovación, la movilidad y la reflexión en el ámbito universitario fueran unas de las piezas clave. La consecución del EEES ha exigido y exige en nuestras universidades, grandes cambios tanto políticos como metodológicos (Álvarez-Rojo et al., 2009). Estos cambios afectan tanto al currículo como a las metodologías. En lo relativo al currículo, resulta fundamental que en los Libros Blancos de las Titulaciones elaborados por la Agencia Nacional de Evaluación de la Calidad y la Acreditación (ANECA), figure la competencia en comunicación oral y escrita en lengua nativa entre las competencias profesionales y destrezas genéricas que debe adquirir el alumnado al finalizar sus futuros estudios de grado, cualesquiera que estos sean. En cuanto a la metodología, el fomento de clases participativas en forma de seminarios, que fomenten el aprendizaje autónomo, el trabajo de grupos reducidos con una metodología plural, debates y exposiciones orales según el Modelo de Bolonia. Todo esto en la línea del establecimiento de un nuevo enfoque de adquisición de competencias; en contraposición a las clases magistrales tradicionales, apunta una vez más hacia la necesidad real de nuestros universitarios por convertirse en comunicadores de excelencia, capaces de construir y emitir mensajes comprensibles y coherentes con las situaciones comunicativas diversas a las que deberán hacer frente.

En este proceso de convergencia europea, tener una buena competencia comunicativa es un aspecto de gran relevancia. Como indican Barrio y Borragán (2011), comunicar con eficacia es importante y facilita el éxito profesional, social y personal. Una buena comunicación permite llegar mejor al otro, y también que la otra persona se sienta entendida. Estas cuestiones son de gran relevancia para el bienestar y el éxito interpersonal y profesional.

Resulta prioritario, por tanto, dotar a docentes (y también a alumnos) de las habilidades y capacidades que les permitan comunicarse en las mejores condiciones posibles. De hecho, varios documentos que se han elaborado en el contexto del Espacio Europeo de Educación Superior ponen de manifiesto la importancia que adquiere la competencia comunicativa. (Ministerio de Educación, Deporte y Cultura 2003, Descriptores de Dublín 2004, Comisión Europea 2004).

Queremos dejar claro que la noción de competencia comunicativa que aquí planteamos trasciende al concepto de competencia lingüística, la cual está limitada a la condición de gramaticalidad. Diversas investigaciones han intentado aislar criterios y condiciones generales para abordar la com- 
petencia comunicativa coincidiendo, en cierta medida, en la distinción de componentes de distinta índole. Concretamente, se distingue entre aspectos cognitivos, afectivos y comportamentales (Vilá, 2003). De esta forma, la competencia comunicativa que tratamos de potenciar es aquella que configura el conjunto de habilidades cognitivas y afectivas que debemos movilizar para manifestar comportamientos apropiados y efectivos en un contexto social, profesional y cultural determinado, favoreciendo un grado de comunicación suficientemente eficaz.

Sin embargo, pese a todos los recientes esfuerzos en el estudio de la habilidad comunicativa, especialmente en la docencia, queda todavía mucho recorrido en el conocimiento de los indicadores y las pautas precisas para el trabajo de esta habilidad entre los docentes, agentes clave en el trabajo con el alumnado y la comunidad universitaria en general. Además, desde nuestra perspectiva, resulta de vital importancia generar instrumentos que permitan reflexionar sobre estos indicadores precisos de la comunicación eficaz. Por ello, es necesario desarrollar instrumentos eficaces de evaluación de la habilidad comunicativa, que sirvan además de elemento de reflexión generalizado entre los docentes universitarios.

\section{METODOLOGÍA}

\section{Participantes}

El presente proyecto ha contado para el análisis de resultados con 156 docentes universitarios, de diferentes perfiles académicos y diferente relación contractual. Todos ellos han participado por iniciativa propia voluntariamente. De ellos, el $57,7 \%$ (90) son hombres y el $42,3 \%$ (66) son mujeres. De la muestra, la mayor parte (el $89,1 \%$ ) tiene entre 30 y 60 años, siendo el $4,5 \%$ menores de 30 años y el 6,4\% mayores de 60 . En relación con el tiempo de experiencia docente nos encontramos con una experiencia docente desigual. De este modo, podemos ver que el $41 \%$ (64 personas) tienen una experiencia de entre 5 y 20 años, en contraste con el 1,9\% (3 personas) con una experiencia de más de 35 años, por lo que los datos obtenidos para esta categoría concreta se consideran irrelevantes. Por otro lado, el 25,6\% (40 personas) tiene menos de 5 años de trayectoria docente, mientras que el $31,4 \%$ (49 personas) ha sido docente durante un periodo de entre 21 y 35 años.

En lo relativo a la categoría profesional, el menor porcentaje de participantes son los profesores ayudantes y ayudantes doctores, contemplando el $5,1 \%$ de la muestra en cada uno de los casos (hay que tener en cuenta que posiblemente sean colectivos más minoritarios). La mayor respuesta se ha encontrado entre los profesores con plena dedicación docente y los profesores asociados, con el $34 \%$ y el $20,5 \%$ respectivamente. Finalmente, destaca el 9,6\% de personas participantes categorizadas en "Otros", de tal forma que no se incluyen en ninguna de las categorías docentes reconocidas por la universidad, como pueden ser becarios de investigación y otros profesionales de la Universidad.

\section{Instrumento}

La herramienta para la Evaluación de la Habilidad Comunicativa Docente es un instrumento realizado para el Aula de Oratoria de la Universidad de Cantabria http://auladeoratoria.unican.es/hcdu12, para la reflexión sobre los puntos fuertes y débiles de esta Habilidad, formado por 61 cuestiones distribuidas en nueve dimensiones:

El mensaje, cuyas cuestiones se centran en la forma y contenido en que se articulan los mensajes que los docentes trasmiten a los alumnos.

Atención, comprensión y feedback, en la que se plantean preguntas relacionadas con el grado en que seguimos el ritmo de los alumnos en relación con la atención y comprensión que muestran.

El lenguaje, con el análisis de la puesta en práctica del lenguaje más adecuado y eficaz para la práctica docente. 
Habla, voz y lenguaje corporal, cuyas cuestiones incluyen aspectos más específicos del uso de las diferentes vías de comunicación (oral y no oral).

Acción/Experimentación para favorecer la comprensión, en la línea de cuestiones relacionadas con la vivencia del aprendizaje.

Inducción al trabajo, al estudio y a la próxima sesión, con la que poder conocer el grado de motivación que se genera en el alumnado.

Participación de los alumnos.

Personalidad, empatía, seducción, optimismo y energía. En esta categoría se abordan cuestiones relativas a la propia manera de ser y estar en el aula y con el alumnado.

Relaciones del profesor con el estudiante, en la que el interés se centra en conocer la percepción de los docentes sobre cómo es la interacción con el alumnado.

En cada una de las dimensiones hay una serie de preguntas a las que cada docente deberá responder de 0 a 3 en función del grado de acuerdo o desacuerdo con las mismas, siendo 0 totalmente en desacuerdo y 3 totalmente de acuerdo.

En función de la puntuación en las respuestas dadas se obtienen las pautas de actuación concretas asociadas a cada cuestión, de forma que el resultado final es personalizado, obteniendo consejos útiles, a través de la Guía de Pautas, para la mejora de aquellas cuestiones en las que de forma personal se haya puntuado más bajo. Además, se puede ver un gráfico con los resultados globales para cada bloque y una valoración general de nuestra comunicación.

\section{RESULTADOS}

A continuación, comentaremos de forma descriptiva algunos de los análisis realizados, con la cautela y la prudencia que conlleva el tamaño de la muestra. Debemos recordar, que el interés fundamental de este instrumento es "mover" al profesorado a una reflexión sobre las cuestiones contempladas en la LUPA y trazar un camino para una posible investigación futura, más que intentar obtener en el momento actual, con la muestra obtenida ningún tipo de datos significativos. Por ello, vamos a realizar unos análisis de una forma general y descriptiva.

Desde esta perspectiva, nos resulta interesante observar (ver figura 1) que la percepción que los docentes tienen en relación con la calidad de los mensajes que utilizan en su docencia es mejor con el paso de los años de experiencia. Si bien es verdad que la percepción es positiva en todas las categorías.

Figura 1. Gráfico de medias de la percepción que los docentes tienen sobre sus mensajes docentes.

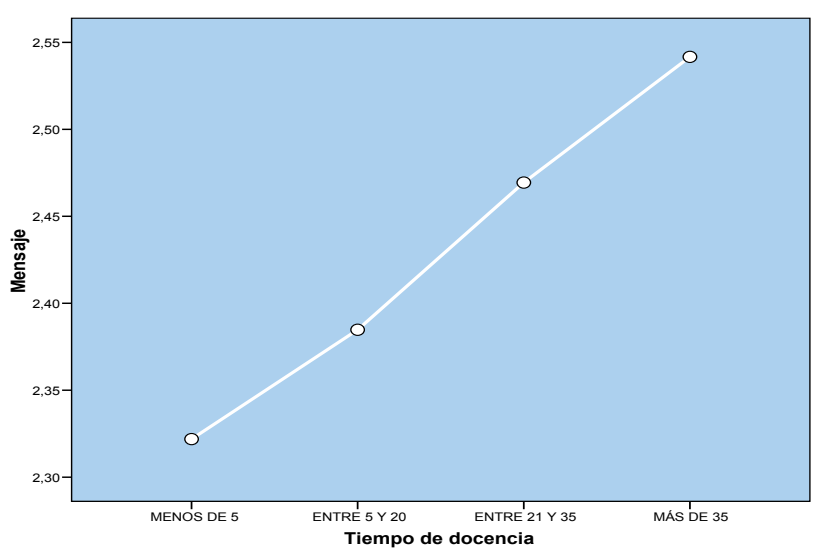


En la misma línea nos encontramos en relación con la dimensión "Atención, comprensión y feedback", en la que nuevamente encontramos una progresión positiva clara en la propia percepción del manejo comunicativo en estos aspectos (ver figura 2). Así, parece que los docentes con mayor experiencia sienten que favorecen en mayor medida la atención y comprensión del alumnado, ofreciéndoles retroalimentación para evitar que desconecten y favorecer su implicación.

Figura 2. Gráfico de medias de la percepción que los docentes tienen sobre la atención, comprensión y feedback docentes.

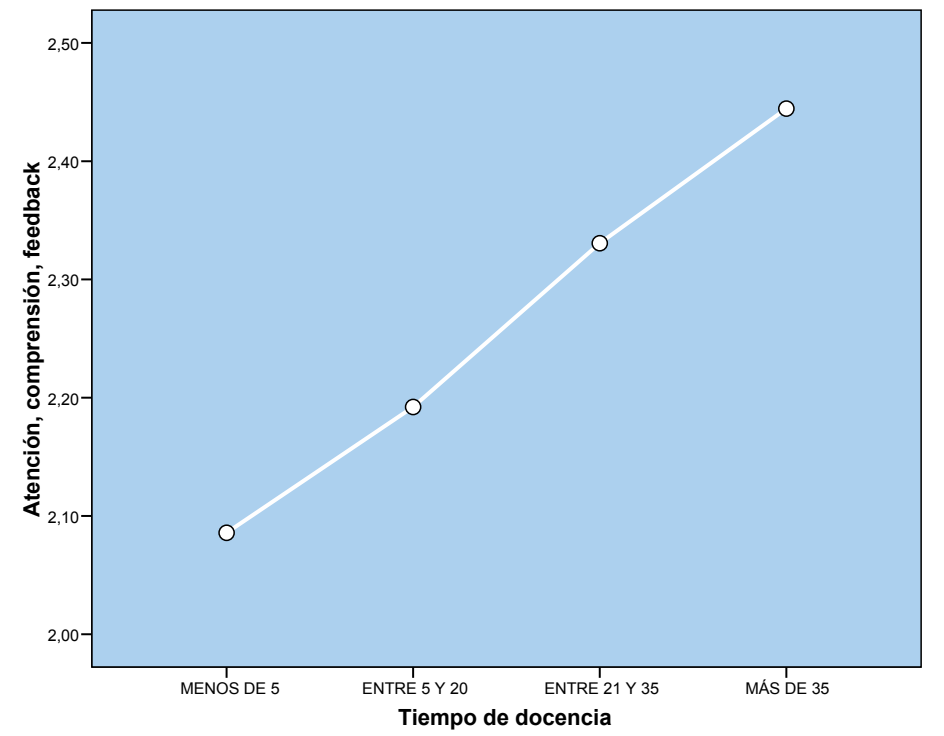

Cuando analizamos la dimensión relacionada con el lenguaje y las posibilidades de expresarnos que nos ofrece, parece que la trayectoria también es, ascendente, de tal modo que a medida que los docentes tienen más experiencia, exponen sentir mejor manejo del lenguaje que utilizan. Aparece un ligerísimo descenso en la puntuación media de las respuestas en el caso de las personas con una experiencia mayor de 35 años. Debemos de recordar que, con una experiencia de más de 35 años, únicamente habían respondido 3 profesores, por lo que los datos obtenidos para esta categoría concreta se consideran irrelevantes. 
Figura 3. Gráfico de medias de la percepción que los docentes tienen sobre el lenguaje docente.

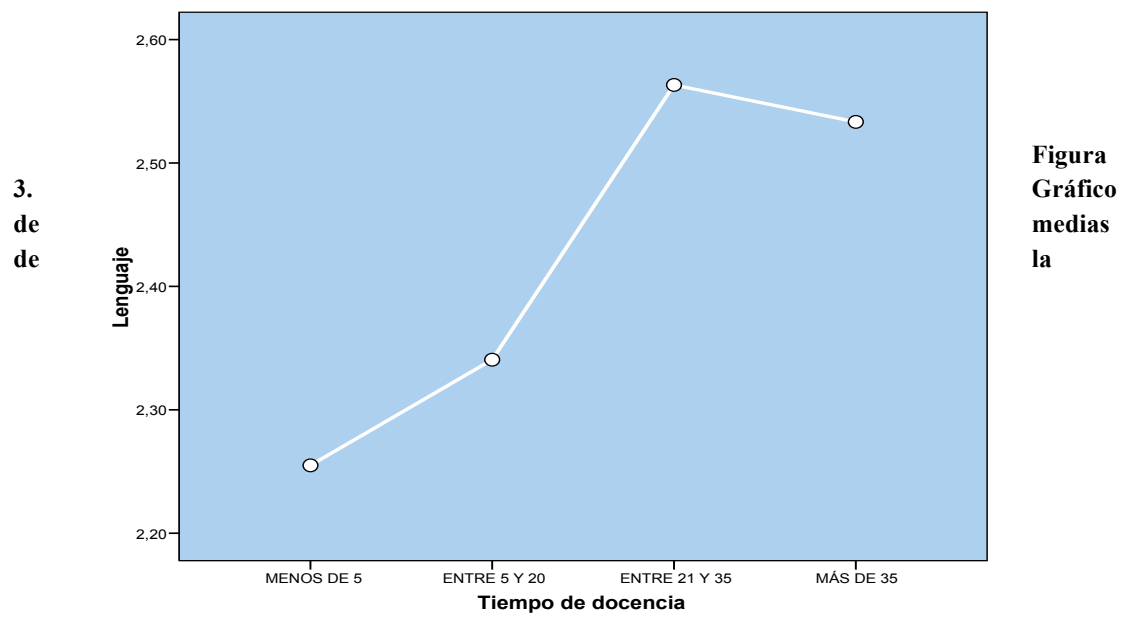

En relación con el buen manejo de su técnica vocal, su habla y su lenguaje corporal, mostrando por término general puntuaciones medias altas y altas (ver figura 4).

Figura 4. Gráfico de medias de la percepción que los docentes tienen sobre el habla, la voz y el lenguaje corporal.

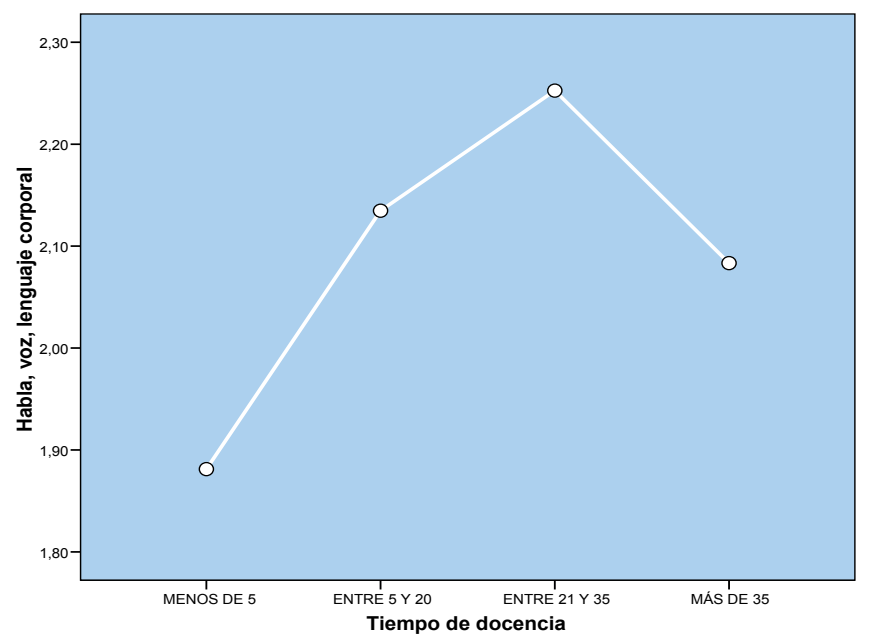

Por otro lado, el patrón de respuesta media es similar en el caso de la dimensión Acción y Experimentación para favorecer la comprensión de lo explicado (ver figura 5), si bien, podemos observar que es en esta dimensión en donde las puntuaciones medias son menores en general en relación con otras dimensiones comunicativas, independientemente de la experiencia docente. 
Figura 5. Gráfico de medias de la percepción que los docentes tienen sobre su Acción y experimentación en la docencia.

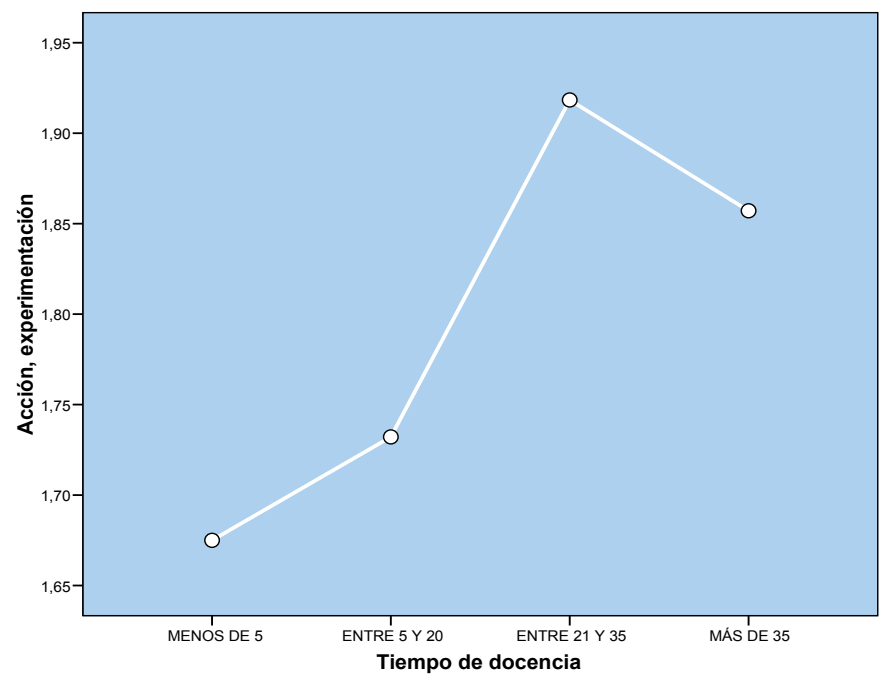

Como podemos ver en la figura 6 , parece que a medida que los docentes adquieren experiencia, se produce una mayor inducción al trabajo y al estudio. Una vez más conviene recordar lo irrelevante que pueda resultar la puntuación del grupo de profesores con más de 35 años de experiencia debido a lo exiguo de la muestra.

Figura 6. Gráfico de medias de la percepción que los docentes tienen sobre la inducción al trabajo, al estudio y a la próxima clase.

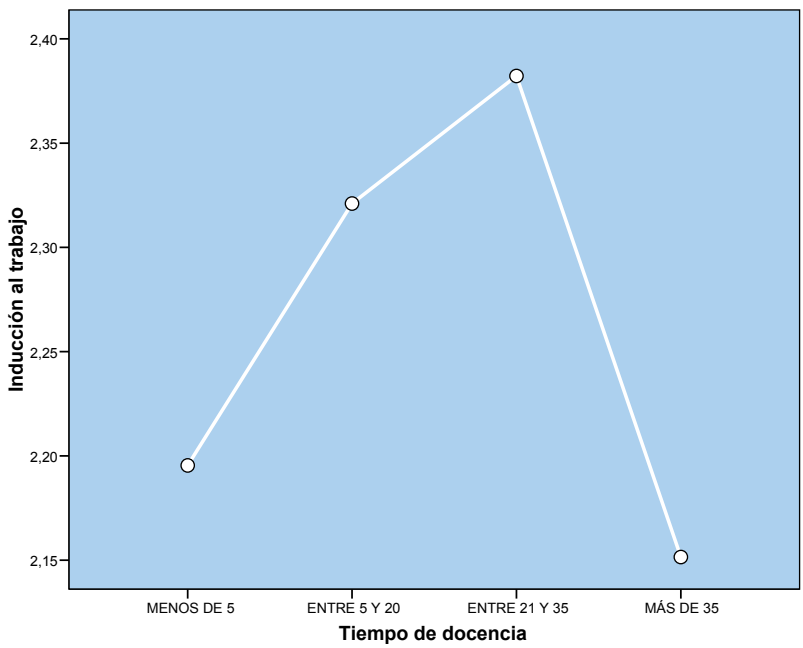


En este nuevo modelo del que hemos hablado en la parte teórica, en el que el alumno asume un mayor protagonismo y se convierte en un agente más activo que nunca, resulta fundamental su participación. En este sentido, las puntuaciones generales en esta dimensión son bastante altas (ver figura 7), especialmente entre los docentes con una experiencia de entre 5 y 20 años, siendo la más baja entre los docentes con menos experiencia y encontrando un ligero, pero progresivo descenso pasados los 20 años de experiencia docente.

Figura 7. Gráfico de medias de la percepción que los docentes tienen sobre la participación del alumnado.

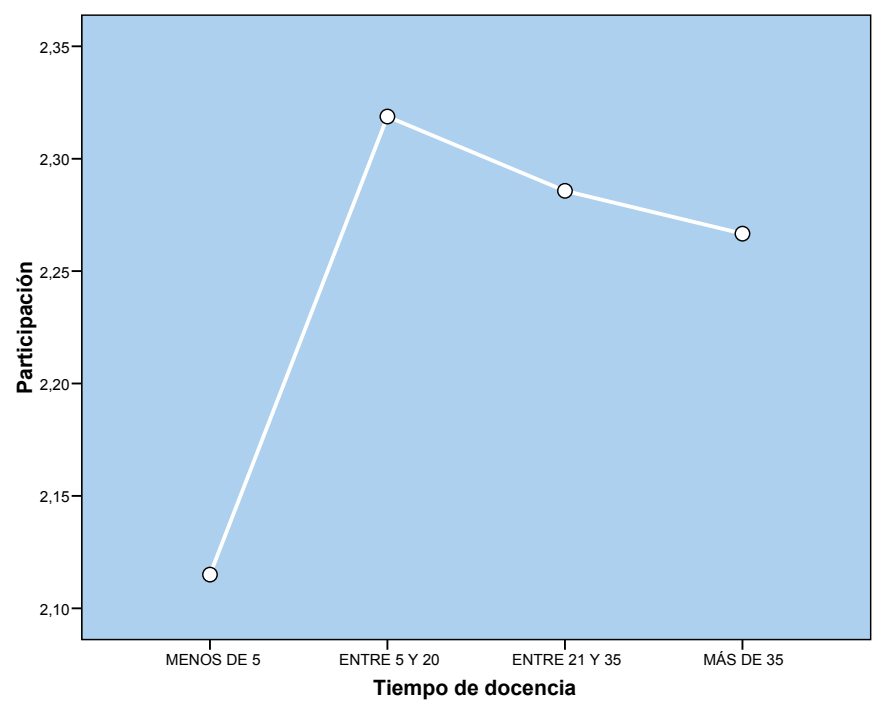

Un resultado diferente y característico es el patrón de respuesta encontrado en la dimensión personalidad, donde se contemplan aspectos personales, de energía, optimismo, etc. como facilitadores de la eficacia comunicativa docente. De este modo no encontramos diferencias en las puntuaciones medias de los docentes hasta los 20 años de experiencia (ver figura 8). Por otro lado, la mejor percepción en cuanto a poner en marcha habilidades de personalidad eficiente en la comunicación la muestran los docentes de entre 21 y 35 años de experiencia docente. El descenso en la puntuación en aquellos docentes con más de 35 años de experiencia habrá que contextualizarlo con los mismos criterios anteriores. 
ANÁLISIS DE LA HABILIDAD COMUNICATIVA DOCENTE Y PAUTAS DE ACTUACIÓN. ATRAER LA ATENCIÓN HABLANDO: UN RETO PARA LA ENSEÑANZA UNIVERSITARIA

Figura 8. Gráfico de medias de la percepción que los docentes tienen sobre la personalidad.

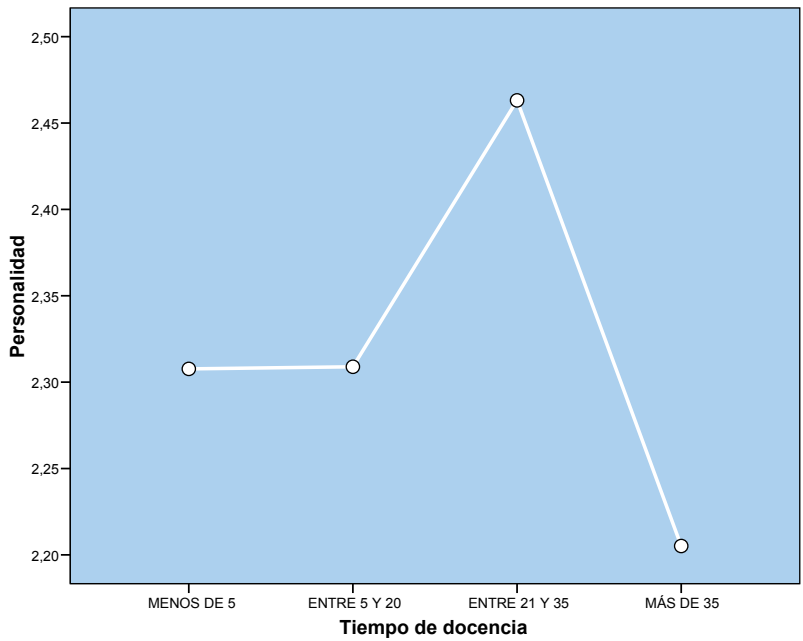

Como en algunas otras dimensiones (ver figura 9), parece que son los docentes con una experiencia media, entre los 5 y 35 años, pero especialmente con una experiencia de entre 21 y 35 años, los que sienten y perciben una mejor relación con sus alumnos.

Figura 9. Gráfico de medias de la percepción que los docentes tienen sobre las relaciones con el alumnado.

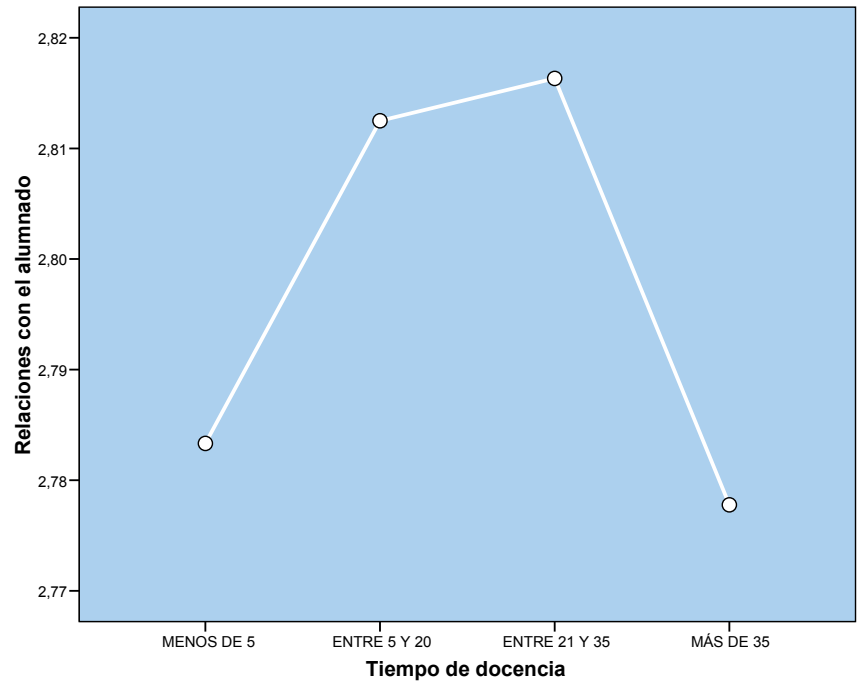


Finalmente, hemos tratado de conocer en qué medida la habilidad comunicativa docente puede ser diferente en varones y mujeres en las diferentes dimensiones. En este sentido, encontramos diferencias en algunas de las dimensiones (ver Tabla 1), resultando curioso que tienen una mejor percepción las mujeres que los varones en todas las dimensiones. Por otro lado, la diferencia más destacable se produce en relación con la dimensión de Acción/Experimentación, siendo este aspecto el único en que la muestra, en este caso los varones, responden por término medio por debajo de la puntuación 2.

De este modo, obtenemos resultados descriptivos que nos permiten analizar estas diferencias en las diferentes áreas (ver Tabla 1).

Tabla 1. Resultados descriptivos en relación con las dimensiones del instrumento para varones y mujeres, así como las diferencias entre ambos.

\begin{tabular}{|c|c|c|c|c|c|c|c|c|c|c|c|c|}
\hline \multirow[t]{2}{*}{$\mathrm{N}=156$} & \multirow[t]{2}{*}{$\begin{array}{c}\text { Rango } \\
\text { de } \\
\text { respuesta }\end{array}$} & \multicolumn{2}{|c|}{ Mínimo } & \multicolumn{2}{|c|}{ Máximo } & \multicolumn{2}{|c|}{ Media (M) } & \multicolumn{2}{|c|}{$\begin{array}{l}\text { Desviación } \\
\text { Típica (DT) }\end{array}$} & \multirow[t]{2}{*}{$\mathrm{F}$} & \multirow[t]{2}{*}{$\mathrm{t}$} & \multirow[t]{2}{*}{$\mathrm{p}$} \\
\hline & & $\mathrm{V}$ & $\mathrm{M}$ & $\mathrm{V}$ & $\mathrm{M}$ & $\mathbf{V}$ & M & $\mathrm{V}$ & $\mathrm{M}$ & & & \\
\hline Mensaje & $0-3$ & 1 & 1 & 3 & 3 & 2,36 & 2,44 & ,41 & 46 & .56 & $-1,05$ & ,29 \\
\hline $\begin{array}{l}\text { Atención, } \\
\text { comprensión, } \\
\text { feedback }\end{array}$ & $0-3$ &, 83 & 1 & 3 & 3 & 2,14 & 2,30 & , 48 & ,48 & .02 & $-1,98$ & ,04 \\
\hline Lenguaje & $0-3$ & 1 & 1 & 3 & 3 & 2,31 & 2,50 & , 44 & ,43 & .08 & 2,63 & .00 \\
\hline $\begin{array}{l}\text { Habla, voz, } \\
\text { lenguaje } \\
\text { corporal }\end{array}$ & $0-3$ & 63 & 1 & 3 & 3 & 2,01 & 2,23 & , 46 & ,47 & .03 & $-2,88$ & .00 \\
\hline $\begin{array}{l}\text { Acción, } \\
\text { experimentación }\end{array}$ & $0-3$ &, 57 & ,29 & 3 & 3 & 1,70 & 2,44 & ,50 & 49 & 7.16 & $-1,96$ & .05 \\
\hline $\begin{array}{l}\text { Inducción al } \\
\text { trabajo }\end{array}$ & $0-3$ & ,67 & ,67 & 3 & 3 & 2,27 & 2,44 & ,47 & .49 & .01 & $-2,29$ & .02 \\
\hline Participación & $0-3$ & 1 & 80 & 3 & 3 & 2,18 & 2,35 & ,48 & ,47 & .10 & -2.14 & .03 \\
\hline Personalidad & $0-3$ & ,85 &, 77 & 3 & 3 & 2,26 & 2,47 & ,44 & 40 & 1.87 & -3.09 & .00 \\
\hline Relaciones & $0-3$ & 1 & ,67 & 3 & 3 & 2,77 & 2,85 & ,37 & ,41 & 1.45 & -1.30 & .19 \\
\hline
\end{tabular}

Finalmente reseñar que los resultados obtenidos a partir de las respuestas de la muestra están condicionados, por un lado, por el número de participantes, puesto que sería conveniente contar con mayor muestra y, por otro lado, a que la muestra hubiese estado repartida de forma similar en las diferentes categorías analizadas (años de experiencia como docente).

\section{CONCLUSIONES}

En relación con la experiencia docente, a la luz de estos datos, parece que la habilidad comunicativa docente de manera general aumenta con la experiencia, al menos esa es la percepción que los propios docentes tienen, si bien habrá que realizar una investigación con muestras más amplias, para poder obtener resultados más precisos y significativos.

Tomado esto en cuenta, podemos concluir de manera general que los componentes de la mues- 


\section{ANÁLISIS DE LA HABILIDAD COMUNICATIVA DOCENTE Y PAUTAS DE ACTUACIÓN. ATRAER LA ATENCIÓN HABLANDO: UN RETO PARA LA ENSEÑANZA UNIVERSITARIA}

tra hacen una valoración positiva de su competencia comunicativa, puesto que las puntuaciones medias en todas las dimensiones son elevadas en relación con el rango de respuesta posible.

Por otro lado, parece que a medida que los docentes cuentan con más experiencia, expresan una mejor habilidad comunicativa. Esto es especialmente claro en relación con el contenido y estructura del mensaje, a la riqueza e idoneidad del lenguaje utilizado, así como a la capacidad de favorecer la comprensión y la atención a través del feedback al alumnado.

Estos datos quizás puedan generar un espacio de reflexión sobre los motivos por los que esto ocurre, contemplando la necesidad de corroborar o refutar estos resultados en una mayor muestra.

En relación con las diferencias entre varones y mujeres en las dimensiones estudiadas, cabe resaltar que son las mujeres las que muestran puntuaciones más altas en todas las dimensiones, siendo la dimensión Acción, experimentación la que muestra mayores diferencias.

Sería importante encontrar quizás el contrapunto de la percepción del alumnado, para acercarnos mejor a la realidad comunicativa del contexto universitario docente.

\section{REFERENCIAS BIBLIOGRÁFICAS}

Abascal, M. D. (2010). Evaluación del uso oral como proyecto de centro. Textos, 53, 48-57.

Álvarez-Rojo, V. et al (2009). Perfiles docentes para el Espacio Europeo de Educación Superior (EEES) en el ámbito universitario español. RELIEVE, 15, 1, 1-18.

Barrio, J.A. y Borragán, A. (2005). El arte de Hablar. Prevenir los problemas de Voz. Madrid. Ministerio de Educación.

Barrio J.A. y Borragán, A. (2011). Cómo atraer la atención hablando. Un reto para la enseñanza. Bordón, 63, 2, 15-25.

Benavides J. (2008). El estado de la comunicación en la enseñanza universitaria: una reflexión sobre el futuro y el pasado inmediato. Revista Comunicación y Pluralismo. Salamanca: Ed. UPS.

Borragán, A, Barrio J.A. y Borragán, V. (2009). El Arte de Hablar. Oratoria Eficaz. Madrid. Ministerio de Educación.

Cifuentes, P. (2008). Panorama de la Educación Superior en Europa en el marco del proceso de Bolonia. En Flecha, J. R. (coord.). El proceso de Bolonia y la Enseñanza Superior en Europa. Ed. Instituto Superior Estudio Europeos.

Comisión Europea (2004). Competencias clave para un aprendizaje a lo largo de la vida. Un marco de referencia europeo. Bruselas: Dirección General de Educación y Cultura. Grupo de trabajo B: Competencias clave.

Declaración de Bolonia (1999). El Espacio Europeo de Educación Superior. Declaración Conjunta de los ministros europeos de educación. Bolonia.

Declaración de la Sorbona (1998). Declaración conjunta para la armonización del diseño del Sistema de Educación Superior Europeo. París.

Descriptores de Dublín (2004). Apéndice 6. Ministry of Science Technology and Innovation.

Ministerio de Educación, Deporte y Cultura (2003). Documento Marco: La integración del sistema universitario español en el Espacio Europeo de Enseñanza Superior. Madrid: Ministerio de Educación, Deporte y Cultura.

Rodriguez, A. (2007). Las competencias en el Espacio Europeo de Educación Superior. Tipologías. Humanismo y trabajo social, 6, 139-153.

Vilà, R. (2003). El desarrollo de la competencia comunicativa intercultural en una sociedad multicultural y plurilingüe: una propuesta de instrumentos para su evaluación. 